\title{
Assessing Multiplex Tiling PCR Sequencing Approaches for Detecting Genomic Variants of SARS-CoV-2 in Municipal Wastewater
}

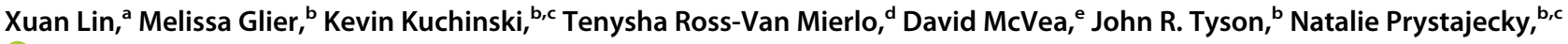 \\ (D) Ryan M. Ziels ${ }^{\mathrm{a}}$ \\ aCivil Engineering, The University of British Columbia, Vancouver, British Columbia, Canada \\ bEnvironmental Microbiology, British Columbia Center for Disease Control Public Health Laboratory, Vancouver, British Columbia, Canada \\ cPathology and Laboratory Medicine, The University of British Columbia, Vancouver, British Columbia, Canada \\ dBiological Sciences, Simon Fraser University, Burnaby, British Columbia, Canada \\ eEnvironmental Health Services, British Columbia Center for Disease Control Public Health Laboratory, Vancouver, British Columbia, Canada
}

ABSTRACT Wastewater-based genomic surveillance of the severe acute respiratory syndrome coronavirus 2 (SARS-CoV-2) virus shows promise to complement genomic epidemiology efforts. Multiplex tiling PCR is a desirable approach for targeted genome sequencing of SARS-CoV-2 in wastewater due to its low cost and rapid turnaround time. However, it is not clear how different multiplex tiling PCR primer schemes or wastewater sample matrices impact the resulting SARS-CoV-2 genome coverage. The objective of this work was to assess the performance of three different multiplex primer schemes, consisting of 150-bp, 400-bp, and 1,200-bp amplicons, as well as two wastewater sample matrices, influent wastewater and primary sludge, for targeted genome sequencing of SARS-CoV-2. Wastewater samples were collected weekly from five municipal wastewater treatment plants (WWTPs) in the Metro Vancouver region of British Columbia, Canada during a period of increased coronavirus disease 19 (COVID-19) case counts from February to April 2021. RNA extracted from clarified influent wastewater provided significantly higher genome coverage (breadth and median depth) than primary sludge samples across all primer schemes. Shorter amplicons appeared to be more resilient to sample RNA degradation but were hindered by greater primer pool complexity in the 150-bp scheme. The identified optimal primer scheme ( $400 \mathrm{bp}$ ) and sample matrix (influent) were capable of detecting the emergence of mutations associated with genomic variants of concern, for which the daily wastewater load significantly correlated with clinical case counts. Taken together, these results provide guidance on best practices for implementing wastewater-based genomic surveillance and demonstrate its ability to inform epidemiology efforts by detecting genomic variants of concern circulating within a geographic region.

IMPORTANCE Monitoring the genomic characteristics of the SARS-CoV-2 virus circulating in a population can shed important insights into epidemiological aspects of the COVID-19 outbreak. Sequencing every clinical patient sample in a highly populous area is a difficult feat, and thus sequencing SARS-CoV-2 RNA in municipal wastewater offers great promise to augment genomic surveillance by characterizing a pooled population sample matrix, particularly during an escalating outbreak. Here, we assess different approaches and sample matrices for rapid targeted genome sequencing of SARS-CoV-2 in municipal wastewater. We demonstrate that the optimal approach is capable of detecting the emergence of SARS-CoV-2 genomic variants of concern, with strong correlations to clinical case data in the province of British Columbia. These results provide guidance on best practices on, as well as further support for,
Citation Lin X, Glier M, Kuchinski K, Ross-Van Mierlo T, McVea D, Tyson JR, Prystajecky N, Ziels RM. 2021. Assessing multiplex tiling PCR sequencing approaches for detecting genomic variants of SARS-COV-2 in municipal wastewater. mSystems 6:e01068-21. https://doi .org/10.1128/mSystems.01068-21.

Editor lleana M. Cristea, Princeton University Copyright $\odot 2021$ Lin et al. This is an openaccess article distributed under the terms of the Creative Commons Attribution 4.0 International license.

Address correspondence to Ryan M. Ziels, ziels@mail.ubc.ca.

Received 24 August 2021

Accepted 22 September 2021

Published 19 October 2021 
the application of wastewater genomic surveillance as a tool to augment current genomic epidemiology efforts.

KEYWORDS COVID-19, RNA, SARS-CoV-2, epidemiology, variants, wastewater, wholegenome sequencing

enomic surveillance of the severe acute respiratory syndrome coronavirus 2 (SARS-CoV-2) virus plays a critical role in tracking its evolution during the current global coronavirus disease 2019 (COVID-19) pandemic (1-3). Recently, several emerging lineages of SARS-CoV-2, so-called variants of concern (VoCs), have been associated with increased levels of transmission (4), disease severity (5), and/or immune escape (6, $7)$. These VoCs have originated from various locations globally $(4,8)$, but they are spreading within new geographic regions due to travel-associated and local transmission (9). Providing rapid detection of $\mathrm{VoC}$ infections within a population could thus help to inform effective public health outbreak mitigation strategies.

Since the SARS-CoV-2 virus is shed in feces during infection (10), viral genome fragments can be detected in municipal wastewater and have been associated with clinical case numbers within contributing regions (11-14). Previous work has demonstrated the potential to sequence SARS-CoV-2 fragments in municipal wastewater and detect single-nucleotide variants (SNVs) that correspond to clinical cases in the contributing sewershed (15-17). As SARS-CoV-2 titers in wastewater are relatively low $(11,13)$, an enrichment step is typically needed prior to sequencing to improve sensitivity (15). The two main approaches for enriching SARS-CoV-2 RNA in wastewater include oligonucleotide-based capture (15) and multiplex tiling PCR-based targeted amplification $(16,17)$. The latter approach is promising for wastewater-based viral genomic surveillance due to its lower reagent cost and the potential to be deployed rapidly and in remote locations (18). An important consideration for applying multiplex tiling PCR is the average amplicon length, as this can impact assay sensitivity in the case of RNA degradation (19). This could be particularly important for its application to wastewater-based epidemiology, as SARS-CoV-2 particles and free RNA can undergo variable levels of degradation $(20,21)$ and may differ based on the type of wastewater sample matrix (e.g., influent versus primary sludge) (22). We therefore hypothesized that there may be an optimal tiling PCR amplicon size and an optimal wastewater sample matrix type that enable adequate genome coverage of SARS-CoV-2 for the identification of genomic VoCs.

SARS-CoV-2 genome coverage is greater with influent wastewater ultrafiltration than with direct sludge extraction and is impacted by multiplex tiling PCR amplicon length. We sequenced a total of 96 wastewater samples collected between 7 February and 18 April 2021 across five municipal WWTPs in Vancouver, Canada, using the following three different primer schemes for multiplex tiling PCR of SARS-CoV-2: Swift Bioscience's 150-bp amplicon scheme ( $n=10$ total, 3 sludge and 7 influent), the Artic 400-bp amplicon scheme (23) ( $n=62$ total, 8 sludge and 54 influent), and the Freed/midnight 1,200-bp amplicon scheme (24) ( $n=24$ total, 4 sludge and 20 influent) (detailed methods are given in Text S1 in the supplemental material). Sludge samples failed to produce libraries with over $32 \%$ breadth of genome coverage across all primer schemes and sample cycle thresholds $\left(C_{T}\right)$ (Fig. 1A to C). Conversely, influent wastewater samples produced libraries that had significantly higher breadth of coverage across all primer schemes $(P<0.01$, Tukey's test; Fig. 1). One possible explanation for this finding could be that the sludge matrix was inhibitory to reverse transcription-PCR (RT-PCR) (11); however, no inhibition of reverse transcription-quantitative PCR on sludge RNA extracts was detected using internal controls (see Text S1 in the supplemental material and Table S2 at https://doi.org/10 $.6084 / \mathrm{m} 9$. figshare.16416528). Another potential reason for the lower genome coverage in sludge was that SARS-CoV-2 was more nonintact or its RNA was more degraded with the direct sludge extraction compared to ultrafiltration of influent wastewater, as has been previously hypothesized (22). A third potential cause of discrepancies in genome coverage between sludge and influent wastewater samples could be higher off-target amplification in sludge extracts. Correspondingly, sample type significantly impacted the fraction of on- 
A 150 bp Amplicons

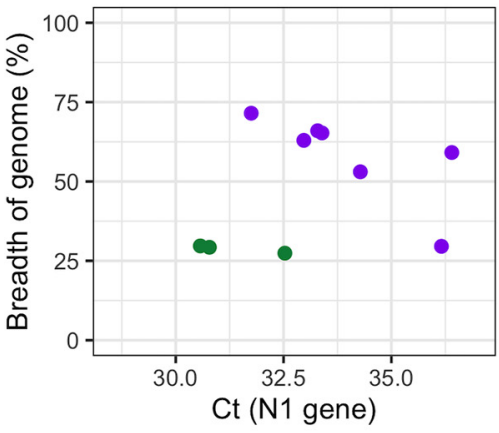

D

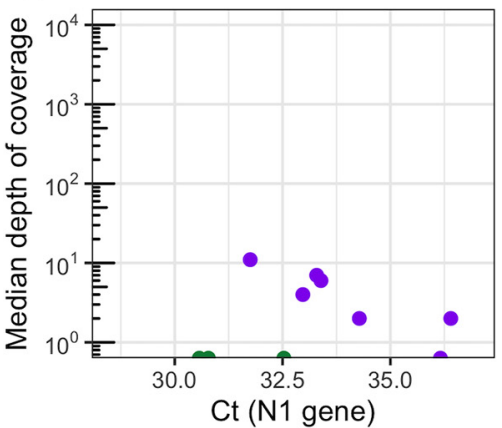

B 400 bp Amplicons

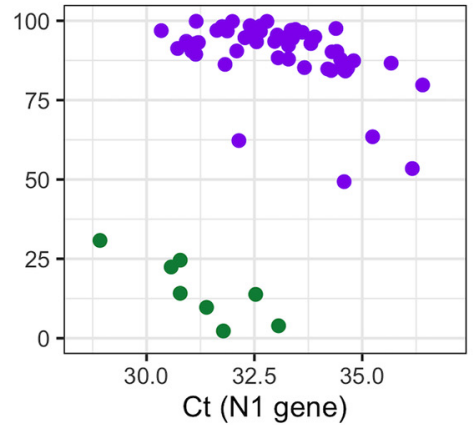

E

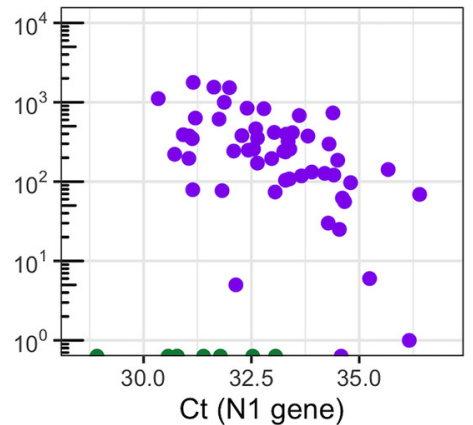

C $\quad 1200$ bp Amplicons

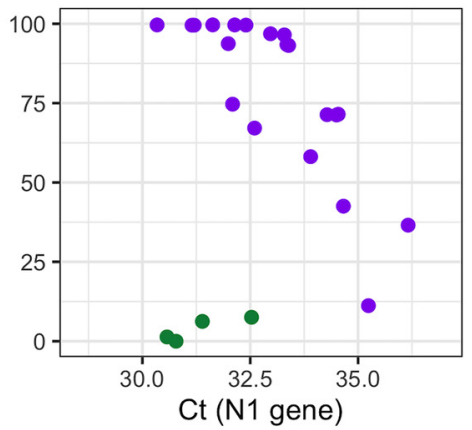

$\mathbf{F}$

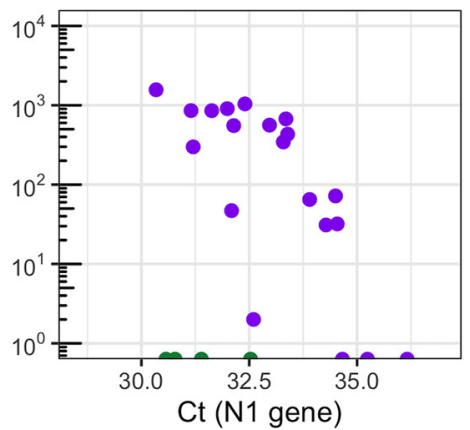

Sample Type: - Primary Sludge • Wastewater

FIG 1 SARS-CoV-2 whole-genome sequencing coverage results from three multiplex tiling PCR primer schemes, including breadth of genome coverage for 150-bp amplicons (A), 400-bp amplicons (B), and 1,200-bp amplicons (C), as well as the median depth of coverage across the genome for 150-bp amplicons (D), 400-bp amplicons (E), and 1,200-bp amplicons (F). The breadth of coverage represents the proportion of nucleotides in the SARS-CoV-2 reference genome (NCBI accession number MN908947.3) that are covered by at least one read, and the median depth of coverage represents the median number of reads mapped at each nucleotide position across the SARS-CoV-2 reference genome. Values are plotted versus the sample cycle threshold $\left(C_{T}\right)$ value for the U.S. CDC N1 assay, measured by reverse transcription-quantitative PCR (RT-qPCR) (see Text S1 in the supplemental material). Data points aligned with the $x$ axis (plots D to F) had values of zero and could not be log transformed.

target reads for all schemes after accounting for $C_{T}$ values $(P<0.01$, two-way analysis of covariance [ANCOVA]), with mean mapping rates of sludge samples being over 100 times lower than that of influent samples $(0.01 \%$ versus $11.3 \%$, respectively; Table S1). Therefore, ultrafiltration of influent wastewater provided more suitable RNA extracts for multiplexed tiling PCR of SARS-CoV-2 than did direct extraction from wastewater sludge, likely due to a combination of greater SARS-CoV-2 RNA degradation and greater off-target amplification in sludge.

If the level of RNA degradation within a wastewater sample impacts the resulting SARS-CoV-2 genome coverage, we would expect to see less of a drop-off in coverage at high $C_{T}$ values for schemes with shorter amplicons. Indeed, we detected a significant effect of amplicon length on the breadth of genome coverage as a function of $C_{T}$ $(P<0.01$, two-way ANCOVA). The median genome coverage with the 150-bp amplicon scheme spanned one order of magnitude within influent wastewater samples that had $C_{T}$ values ranging from 31 to 37 (Fig. 1D), while those from the 400-bp and 1,200-bp schemes spanned 3.2 and 3.0 orders of magnitude, respectively (Fig. $1 \mathrm{E}$ and F). Improvements with the 400-bp scheme versus the 1,200-bp scheme were marginal, yet $83 \%$ of paired influent samples with $C_{T}$ values over 32.5 (10 of 12) showed higher breadth of coverage with the 400-bp scheme (Fig. S1). Thus, shorter amplicon schemes may be more robust to sample RNA degradation at higher $C_{T}$ values. However, there was a trade-off between amplicon length and genome coverage, as the magnitudes of the median genome coverage and breadth of coverage obtained with the 150-bp scheme and influent samples were significantly lower than those obtained with the 

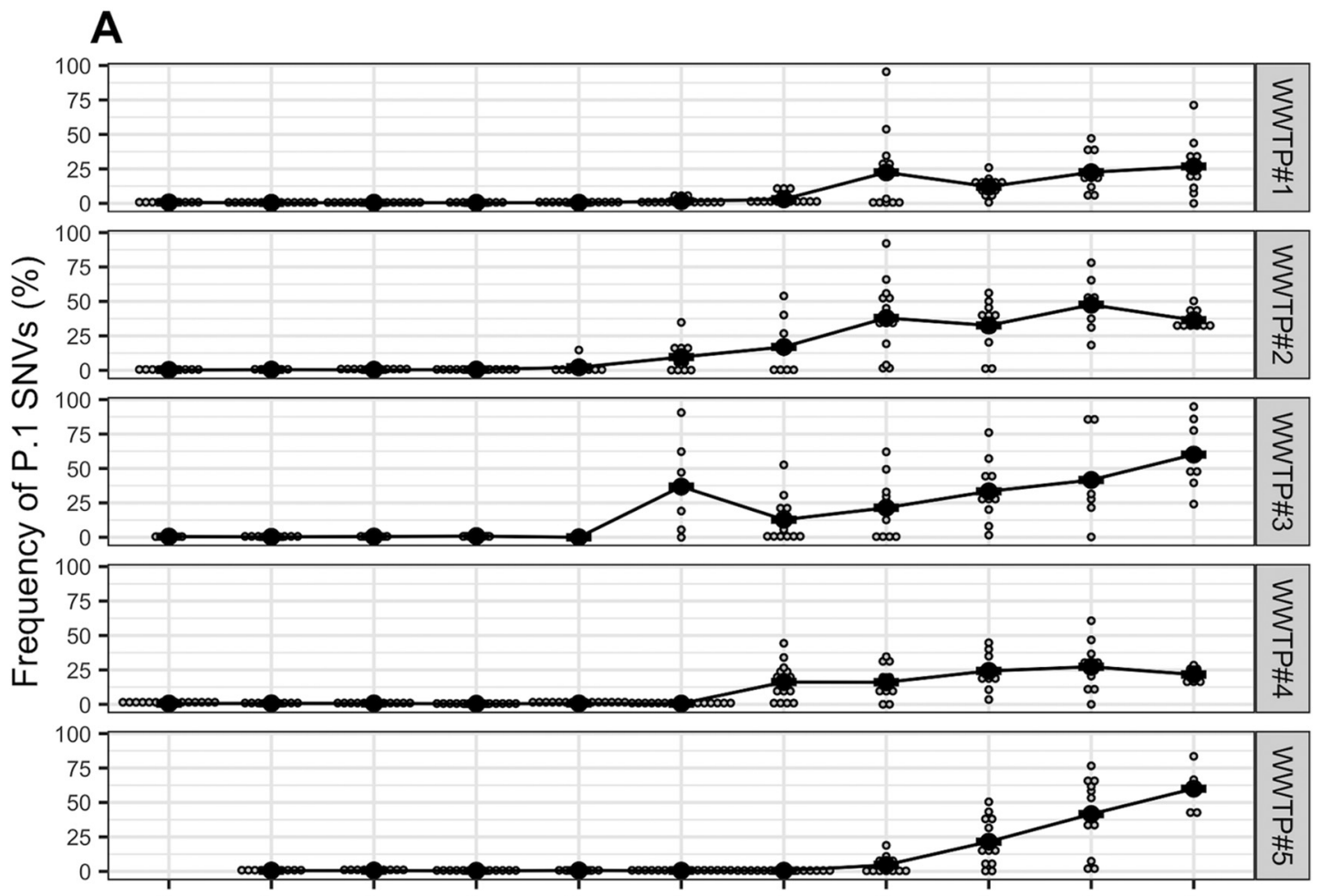

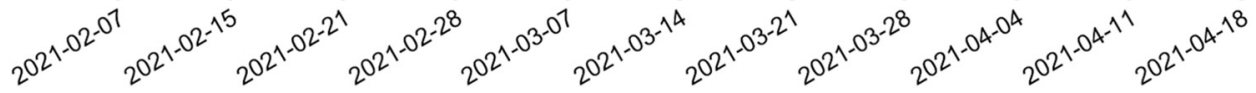

\section{Sample Date}

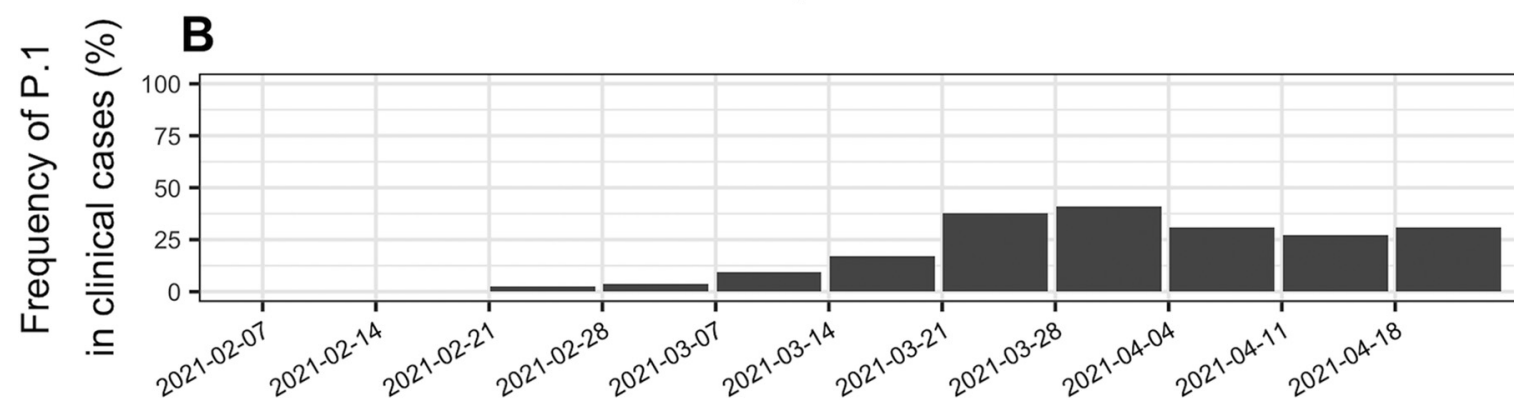

Epi Week

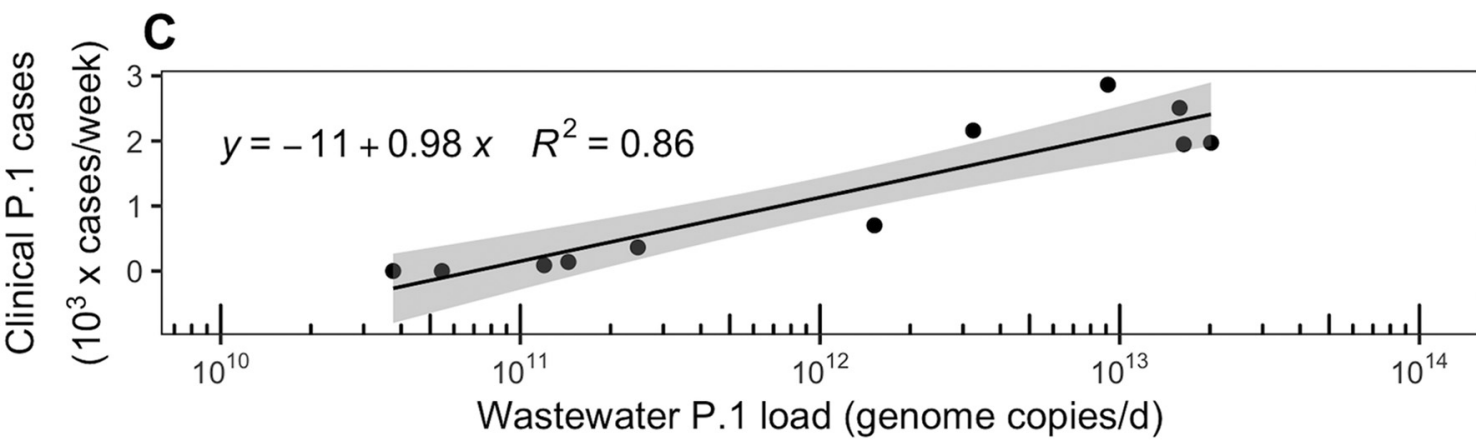

FIG 2 (A) Frequency of single-nucleotide variants (SNVs) associated with the P.1 lineage of SARS-CoV-2 within influent wastewater samples from five wastewater treatment plants in Vancouver, British Columbia (BC), from 7 February to 18 April 2021 . Smaller gray dots represent the frequency of individual variant of concern (VoC)-associated SNVs on the sample dates, while the larger black points represent the mean across all detected VoC-associated SNVs. Only genome positions with a read coverage over 50 are included in SNV frequency calculations. The VoC-associated SNVs are described in Text S1 in the supplemental material and provided in Table S3 at https://doi.org/10.6084/m9.figshare.16416528. (B) Frequency of the P.1 lineage in clinical COVID-19 patient cases in the province of $\mathrm{BC}$, Canada, over the study period. The frequencies in clinical patient cases correspond to average values detected over an epidemiology (epi) week and were adapted from reference 25. (C) Correlation between the wastewater cumulative daily load of 
400-bp scheme ( $P=0.022$ and $5.0 \times 10^{-9}$, respectively; Tukey's test). This result likely cannot be attributed to library preparation and/or the sequencing platform, as we found no significant difference in the breadth of genome coverage obtained on a subset of 10 400-bp amplicon samples sequenced with both Illumina MiSeq and Oxford Nanopore Technologies MinION platforms ( $P=0.5$, Tukey's test; Fig. S2). The lower breadth of coverage observed with the 150-bp scheme could thus have been caused by more primer-primer interactions with a larger number of primers or by the characteristics of the primer pool design (19). Such effects were also indicated by sequencing a synthetic SARS-CoV-2 RNA genome as a positive control, as the 150-bp primer scheme showed more uneven coverage across the genome compared to that of the 400-bp and 1,200-bp schemes (Fig. S3). Therefore, the 400-bp primer scheme appears to strike a balance between resilience to sample RNA degradation and mitigation of issues around primer pool complexity and multiplex amplicon balancing.

SARS-CoV-2 whole-genome sequencing from wastewater captures the emergence of genomic variants in a geographic region. The sequence data produced via the 400-bp primer scheme and influent wastewater samples was used to measure the frequency of VoC-associated SNVs (see Table S3 at https://doi.org/10.6084/m9 .figshare.16416528) across the five WWTPs over the study period. SNVs associated with the VoC lineages B.1.1.7 and P.1 both increased to a maximum mean frequency of $60 \%$ across all WWTPs (Fig. 2A and Fig. S4 to S6), while the frequency of B.1.351 did not substantially increase (Fig. S7). These findings align with the results of clinical screening and sequencing of patient samples over the same period within the province of British Columbia, during which P.1 and B.1.1.7 became the dominant lineages, while B.1.351 did not appreciably spread (25) (Fig. 2B and Fig. S5 and S7). At the time of publishing, $\mathrm{VoC}$ frequency data for clinical cases was only available at the provincial level, yet the health service areas corresponding to the 5 WWTP sewersheds accounted for $74 \%$ of total cases in the province during the study period (25). The log-transformed, flow-normalized daily loads of P.1 and B.1.1.7 across all WWTPs (in genome copies/day) were strongly correlated with clinical case counts of those lineages within the province for the corresponding epidemiological weeks $\left(R^{2}=\right.$ 0.86 and 0.85 , respectively; Fig. $2 C$ and Fig. S5). The log-transformed mean frequencies of VoC-associated SNVs in wastewater were also significantly correlated with that of VoC clinical case counts within the region ( $P<0.01$; Fig. S8). Therefore, the frequency of VoC-associated SNVs within influent wastewater measured with multiplex tiling PCR is a suitable measure to monitor community transmission of genomic variants within a sewershed. The onset of P.1- and B.1.1.7-associated SNVs within influent wastewater followed different patterns for the five WWTPs, providing additional support that wastewater SARS-CoV-2 sequencing can illuminate localized spread of genomic variants on a regional scale $(15,17)$. The rapid turnaround time (here, $\sim 3$ days from sampling to data generation), low capital cost, and high portability of nanopore sequencing combined with highly multiplexed tiling PCR for SARS-CoV-2 sequencing of wastewater shows great promise to complement genomic epidemiology efforts during the COVID-19 pandemic by detecting the emergence of $\mathrm{VoC}$ within a pooled population sample.

Data availability. The raw reads associated with all samples are available in the Sequence Read Archive under BioProject accession number PRJNA731975. The accession numbers for each sample are also provided in Table S1 in the supplemental material, along with the sample metadata.

FIG 2 Legend (Continued)

P.1 genomes summed across all five wastewater treatment plants (WWTPs) and the total P.1 clinical cases in the province of BC observed within the same epidemiological week. The wastewater P.1 daily load (genome copies/day) was approximated by normalizing copies of the SARS-CoV-2 N1 gene (copies/liter) by daily flow rates (liters/day) to obtain N1 loads (copies/day) for all WWTPs and multiplying those by the mean frequency of P.1-associated SNVs in each WWTP across all sample dates. For each date, the cumulative P.1 daily load was determined by summing the P.1 loads across all five WWTPs. The P.1 clinical case counts by week were estimated from reference 25 by multiplying total provincial COVID-19 case counts by the frequency of P.1 in clinical provincial cases. 


\author{
SUPPLEMENTAL MATERIAL \\ Supplemental material is available online only. \\ TEXT S1, PDF file, $0.02 \mathrm{MB}$. \\ FIG S1, PDF file, $0.3 \mathrm{MB}$. \\ FIG S2, PDF file, $0.1 \mathrm{MB}$. \\ FIG S3, PDF file, $0.4 \mathrm{MB}$. \\ FIG S4, PDF file, $0.01 \mathrm{MB}$. \\ FIG S5, PDF file, $0.4 \mathrm{MB}$. \\ FIG S6, PDF file, $0.01 \mathrm{MB}$. \\ FIG S7, PDF file, $0.5 \mathrm{MB}$. \\ FIG S8, PDF file, $0.01 \mathrm{MB}$. \\ TABLE S1, DOCX file, $0.04 \mathrm{MB}$.
}

\title{
ACKNOWLEDGMENTS
}

We thank Farida Bishay, Rob MacArthur, Daisy Espinosa, Alvin Louie, and the entire Metro Vancouver Environmental Management \& Quality Control WWTP Laboratory Staff for collecting and delivering wastewater samples for this study and providing sample metadata. We also thank Ziwen Ran for help with method development and Matthias Krushel for help with processing wastewater samples. We also thank the Molecular and Microbial Genomics and Environmental Microbiology Laboratories at the BCCDC Public Health Laboratory for materials and access to testing equipment and the BCCDC and BC Regional Health Authorities for publicly sharing data on clinical case counts and variants of concern.

This work was funded by the Natural Sciences and Engineering Research Council of Canada (NSERC; Alliance Grant ALLRP 554612-20), the BCCDC Foundation, Metro Vancouver, and Innovate BC.

\section{REFERENCES}

1. Rambaut A, Holmes EC, O'Toole Á, Hill V, McCrone JT, Ruis C, du Plessis L, Pybus OG. 2020. A dynamic nomenclature proposal for SARS-CoV-2 lineages to assist genomic epidemiology. 11. Nat Microbiol 5:1403-1407. https://doi.org/10.1038/s41564-020-0770-5.

2. Bedford T, Greninger AL, Roychoudhury $P$, Starita $L M$, Famulare $M$, Huang M-L, Nalla A, Pepper G, Reinhardt A, Xie H, Shrestha L, Nguyen TN, Adler A, Brandstetter E, Cho S, Giroux D, Han PD, Fay K, Frazar CD, Ilcisin M, Lacombe K, Lee J, Kiavand A, Richardson M, Sibley TR, Truong M, Wolf CR, Nickerson DA, Rieder MJ, Englund JA, Hadfield J, Hodcroft EB, Huddleston J, Moncla LH, Müller NF, Neher RA, Deng X, Gu W, Federman S, Chiu C, Duchin JS, Gautom R, Melly G, Hiatt B, Dykema P, Lindquist S, Queen K, Tao Y, Uehara A, Tong S, Seattle Flu Study Investigators, et al. 2020. Cryptic transmission of SARS-CoV-2 in Washington state. Science 370:571-575. https://doi.org/10.1126/science.abc0523.

3. Candido DS, Claro IM, de Jesus JG, Souza WM, Moreira FRR, Dellicour S, Mellan TA, du Plessis L, Pereira RHM, Sales FCS, Manuli ER, Thézé J, Almeida L, Menezes MT, Voloch CM, Fumagalli MJ, Coletti TM, da Silva CAM, Ramundo MS, Amorim MR, Hoeltgebaum HH, Mishra S, Gill MS, Carvalho LM, Buss LF, Prete CA, Ashworth J, Nakaya HI, Peixoto PS, Brady OJ, Nicholls SM, Tanuri A, Rossi ÁD, Braga CKV, Gerber AL, de Guimarães APC, Gaburo N, Alencar CS, Ferreira ACS, Lima CX, Levi JE, Granato C, Ferreira GM, Francisco RS, Granja F, Garcia MT, Moretti ML, Perroud MW, Castiñeiras TMPP, Lazari CS, Hill SC, de Santos AAS, Simeoni CL, Forato J, Sposito AC, Schreiber AZ, Santos MNN, Brazil-UK Centre for Arbovirus Discovery, Diagnosis, Genomics and Epidemiology (CADDE) Genomic Network, et al. 2020. Evolution and epidemic spread of SARS-CoV-2 in Brazil. Science 369:1255-1260. https://doi.org/10.1126/science.abd2161.

4. Davies NG, Abbott $S$, Barnard RC, Jarvis Cl, Kucharski AJ, Munday JD, Pearson CAB, Russell TW, Tully DC, Washburne AD, Wenseleers T, Gimma A, Waites W, Wong KLM, van Zandvoort K, Silverman JD, Diaz-Ordaz K, Keogh R, Eggo RM, Funk S, Jit M, Atkins KE, Edmunds WJ, CMMID COVID19 Working Group, COVID-19 Genomics UK (COG-UK) Consortium. 2021. Estimated transmissibility and impact of SARS-CoV-2 lineage B.1.1.7 in England. Science 372:eabg3055. https://doi.org/10.1126/ science.abg3055.
5. Davies NG, Jarvis Cl, Edmunds WJ, Jewell NP, Diaz-Ordaz K, Keogh RH. 2021. Increased mortality in community-tested cases of SARS-CoV-2 lineage B.1.1.7. Nature 593:270-274. https://doi.org/10.1038/s41586-021 -03426-1.

6. Wibmer $C K$, Ayres $F$, Hermanus $T$, Madzivhandila $M$, Kgagudi $P$, Oosthuysen B, Lambson BE, de Oliveira T, Vermeulen M, van der Berg K, Rossouw T, Boswell M, Ueckermann V, Meiring $S$, von Gottberg A, Cohen C, Morris L, Bhiman JN, Moore PL. 2021. SARS-CoV-2 501Y.V2 escapes neutralization by South African COVID-19 donor plasma. 4. Nat Med 27: 622-625. https://doi.org/10.1038/s41591-021-01285-x.

7. Wang P, Nair MS, Liu L, Iketani S, Luo Y, Guo Y, Wang M, Yu J, Zhang B, Kwong PD, Graham BS, Mascola JR, Chang JY, Yin MT, Sobieszczyk M, Kyratsous CA, Shapiro L, Sheng Z, Huang Y, Ho DD. 2021. Antibody resistance of SARS-CoV-2 variants B.1.351 and B.1.1.7. Nature 593:130-135. https://doi.org/10.1038/s41586-021-03398-2.

8. Tegally $\mathrm{H}$, Wilkinson $\mathrm{E}$, Giovanetti M, Iranzadeh A, Fonseca V, Giandhari J, Doolabh D, Pillay S, San EJ, Msomi N, Mlisana K, von Gottberg A, Walaza S, Allam M, Ismail A, Mohale T, Glass AJ, Engelbrecht S, Zyl GV, Preiser W, Petruccione F, Sigal A, Hardie D, Marais G, Hsiao M, Korsman S, Davies M-A, Tyers L, Mudau I, York D, Maslo C, Goedhals D, Abrahams S, Laguda-Akingba O, Alisoltani-Dehkordi A, Godzik A, Wibmer CK, Sewell BT, Lourenço J, Alcantara LCJ, Pond SLK, Weaver S, Martin D, Lessells RJ, Bhiman JN, Williamson C, de Oliveira T. 2020. Emergence and rapid spread of a new severe acute respiratory syndrome-related coronavirus 2 (SARS-CoV-2) lineage with multiple spike mutations in South Africa. medRxiv https://doi.org/ 10.1101/2020.12.21.20248640.

9. Washington NL, Gangavarapu K, Zeller M, Bolze A, Cirulli ET, Schiabor Barrett KM, Larsen BB, Anderson C, White S, Cassens T, Jacobs S, Levan G, Nguyen J, Ramirez JM, Rivera-Garcia C, Sandoval E, Wang X, Wong D, Spencer E, Robles-Sikisaka R, Kurzban E, Hughes LD, Deng X, Wang C, Servellita V, Valentine H, De Hoff P, Seaver P, Sathe S, Gietzen K, Sickler B, Antico J, Hoon K, Liu J, Harding A, Bakhtar O, Basler T, Austin B, MacCannell D, Isaksson M, Febbo PG, Becker D, Laurent M, McDonald E, Yeo GW, Knight R, Laurent LC, de Feo E, Worobey M, Chiu CY, et al. 2021. Emergence and 
rapid transmission of SARS-CoV-2 B.1.1.7 in the United States. Cell 184: 2587-2594.e7. https://doi.org/10.1016/j.cell.2021.03.052.

10. Pan Y, Zhang D, Yang P, Poon LLM, Wang Q. 2020. Viral load of SARS-CoV2 in clinical samples. Lancet Infect Dis 20:411-412. https://doi.org/10 .1016/S1473-3099(20)30113-4.

11. D'Aoust PM, Mercier E, Montpetit D, Jia J-J, Alexandrov I, Neault N, Baig AT, Mayne J, Zhang X, Alain T, Langlois M-A, Servos MR, MacKenzie M, Figeys D, Mackenzie AE, Graber TE, Delatolla R. 2021. Quantitative analysis of SARS-CoV-2 RNA from wastewater solids in communities with low COVID-19 incidence and prevalence. Water Res 188:116560. https://doi .org/10.1016/j.watres.2020.116560.

12. Wolfe MK, Archana A, Catoe D, Coffman MM, Dorevich S, Graham KE, Kim S, Grijalva LM, Roldan-Hernandez L, Silverman Al, Sinnott-Armstrong N, Vugia DJ, Yu AT, Zambrana W, Wigginton KR, Boehm AB. 2021. Scaling of SARS-CoV-2 RNA in settled solids from multiple wastewater treatment plants to compare incidence rates of laboratory-confirmed COVID-19 in their sewersheds. Environ Sci Technol Lett 8:398-404. https://doi.org/10 .1021/acs.estlett.1c00184.

13. Peccia J, Zulli A, Brackney DE, Grubaugh ND, Kaplan EH, CasanovasMassana A, Ko Al, Malik AA, Wang D, Wang M, Warren JL, Weinberger DM, Arnold W, Omer SB. 2020. Measurement of SARS-CoV-2 RNA in wastewater tracks community infection dynamics. 10. Nat Biotechnol 38: 1164-1167. https://doi.org/10.1038/s41587-020-0684-z.

14. Wu F, Zhang J, Xiao A, Gu X, Lee WL, Armas F, Kauffman K, Hanage W, Matus M, Ghaeli N, Endo N, Duvallet C, Poyet M, Moniz K, Washburne AD, Erickson TB, Chai PR, Thompson J, Alm EJ. 2020. SARS-CoV-2 titers in wastewater are higher than expected from clinically confirmed cases. mSystems 5:e00614-20. https://doi.org/10.1128/mSystems.00614-20.

15. Crits-Christoph A, Kantor RS, Olm MR, Whitney ON, Al-Shayeb B, Lou YC, Flamholz A, Kennedy LC, Greenwald H, Hinkle A, Hetzel J, Spitzer S, Koble J, Tan A, Hyde F, Schroth G, Kuersten S, Banfield JF, Nelson KL. 2021. Genome sequencing of sewage detects regionally prevalent SARS-CoV-2 variants. $\mathrm{mBio}$ 12:e02703-20.

16. Nemudryi A, Nemudraia A, Wiegand T, Surya K, Buyukyoruk M, Cicha $C$, Vanderwood KK, Wilkinson R, Wiedenheft B. 2020. Temporal detection and phylogenetic assessment of SARS-CoV-2 in municipal wastewater. Cell Rep Med 1:100098. https://doi.org/10.1016/j.xcrm.2020.100098.

17. Izquierdo-Lara R, Elsinga G, Heijnen L, Munnink BBO, Schapendonk CME, Nieuwenhuijse D, Kon M, Lu L, Aarestrup FM, Lycett S, Medema G, Koopmans MPG, de Graaf M. 2021. Monitoring SARS-CoV-2 circulation and diversity through community wastewater sequencing, the Netherlands and Belgium. Emerg Infect Dis 27:1405-1415. https://doi.org/10 .3201/eid2705.204410.
18. Quick J, Loman NJ, Duraffour S, Simpson JT, Severi E, Cowley L, Bore JA, Koundouno R, Dudas G, Mikhail A, Ouédraogo N, Afrough B, Bah A, Baum $\mathrm{JH}$, Becker-Ziaja B, Boettcher J-P, Cabeza-Cabrerizo M, Camino-Sanchez A, Carter LL, Doerrbecker J, Enkirch T, Dorival IGG, Hetzelt N, Hinzmann J, Holm T, Kafetzopoulou LE, Koropogui M, Kosgey A, Kuisma E, Logue $\mathrm{CH}_{\text {, }}$ Mazzarelli A, Meisel S, Mertens M, Michel J, Ngabo D, Nitzsche K, Pallash E, Patrono LV, Portmann J, Repits JG, Rickett NY, Sachse A, Singethan K, Vitoriano I, Yemanaberhan RL, Zekeng EG, Trina R, Bello A, Sall AA, Faye $\mathrm{O}$, et al. 2016. Real-time, portable genome sequencing for Ebola surveillance. Nature 530:228-232. https://doi.org/10.1038/nature16996.

19. Quick J, Grubaugh ND, Pullan ST, Claro IM, Smith AD, Gangavarapu K, Oliveira G, Robles-Sikisaka R, Rogers TF, Beutler NA, Burton DR, LewisXimenez LL, de Jesus JG, Giovanetti M, Hill SC, Black A, Bedford T, Carroll MW, Nunes M, Alcantara LC, Jr, Sabino EC, Baylis SA, Faria NR, Loose M, Simpson JT, Pybus OG, Andersen KG, Loman NJ. 2017. Multiplex PCR method for MinION and Illumina sequencing of Zika and other virus genomes directly from clinical samples. Nat Protoc 12:1261-1276. https:// doi.org/10.1038/nprot.2017.066.

20. Bivins A, Greaves J, Fischer R, Yinda KC, Ahmed W, Kitajima M, Munster VJ, Bibby K. 2020. Persistence of SARS-CoV-2 in water and wastewater. Environ Sci Technol Lett 7:937-942. https://doi.org/10.1021/acs.estlett $.0 \mathrm{c00730.}$

21. Wurtzer $S$, Waldman $P$, Ferrier-Rembert A, Frenois-Veyrat G, Mouchel JM, Boni M, Maday Y, Marechal V, Moulin L. 2021. Several forms of SARS-CoV2 RNA can be detected in wastewaters: implication for wastewater-based epidemiology and risk assessment. Water Res 198:117183. https://doi .org/10.1016/j.watres.2021.117183.

22. Kantor RS, Nelson KL, Greenwald HD, Kennedy LC. 2021. Challenges in measuring the recovery of SARS-CoV-2 from wastewater. Environ Sci Technol 55:3514-3519. https://doi.org/10.1021/acs.est.0c08210.

23. Tyson JR, James P, Stoddart D, Sparks N, Wickenhagen A, Hall G, Choi JH, Lapointe H, Kamelian K, Smith AD, Prystajecky N, Goodfellow I, Wilson SJ, Harrigan R, Snutch TP, Loman NJ, Quick J. 2020. Improvements to the ARTIC multiplex PCR method for SARS-CoV-2 genome sequencing using nanopore. bioRxiv https://doi.org/10.1101/2020.09.04.283077.

24. Freed NE, VIková M, Faisal MB, Silander OK. 2020. Rapid and inexpensive whole-genome sequencing of SARS-CoV-2 using 1200 bp tiled amplicons and Oxford Nanopore rapid barcoding. Biol Methods Protoc 5:bpaa014. https://doi.org/10.1093/biomethods/bpaa014.

25. BC Center for Disease Control. 2021. Weekly update on variants of concern (VOC), May 6, 2021. 